\title{
Erratum to: Turing instabilities in a mathematical model for signaling networks
}

\author{
Andreas Rätz • Matthias Röger
}

Published online: 16 December 2012

(C) Springer-Verlag Berlin Heidelberg 2012

\section{Erratum to: J Math Biol (2012) 65:1215-1244 DOI 10.1007/s00285-011-0495-4}

The authors would like to correct an error in the content of Theorem 4.1 pertaining to the original publication of the article.

In Sect. 4 , the authors consider a spatially homogeneous stationary point $\left(u_{*}, v_{*}\right)$ of the non-local two-variable reaction-diffusion system

$$
\begin{aligned}
\partial_{t} u & =\Delta_{\Gamma} u+\gamma f(u, v), \\
\partial_{t} v & =\Delta_{\Gamma} v+\gamma(-f(u, v)+q(u+v, v, V[u+v]))
\end{aligned}
$$

and evaluate the possibility of a diffusive instability in $\left(u_{*}, v_{*}\right)$. In Theorem 4.1 of the original publication a non-existence result of Turing instabilities is stated. In the proof, a wrong characterization of Turing instability is used: though the condition is sufficient, in general it is not necessary for a non-local two-variable system of the form (1), (2).

In order to correct the statement, an extra condition has to be assumed [see (5) below]. This yields the following, weaker result.

The online version of the original article can be found under doi:10.1007/s00285-011-0495-4.

A. Rätz · M. Röger $(\varangle)$

Technische Universität Dortmund, Dortmund, Germany

e-mail: matthias.roeger@tu-dortmund.de

A. Rätz

e-mail: andreas.raetz@tu-dortmund.de 
Theorem 4.1 Assume that $\left(u_{*}, v_{*}\right)$ is of activator-substrate depletion type, that is

$$
\begin{aligned}
\partial_{u} f\left(u_{*}, v_{*}\right)>0, & \partial_{v} f\left(u_{*}, v_{*}\right)>0, \\
\partial_{u} g_{0}\left(u_{*}, v_{*}\right)<0, & \partial_{v} g_{0}\left(u_{*}, v_{*}\right)<0,
\end{aligned}
$$

and assume in addition that

$$
\partial_{u} f\left(u_{*}, v_{*}\right)\left(\partial_{1} q+\partial_{2} q\right)\left(u_{*}, v_{*}\right)-\partial_{v} f\left(u_{*}, v_{*}\right) \partial_{1} q\left(u_{*}, v_{*}\right) \geq 0 .
$$

Then no Turing type instability of (1), (2) exists in $\left(u_{*}, v_{*}\right)$.

Proof Under the additional assumption (5) the usual conditions for a Turing instability apply and the arguments in the proof of Theorem 4.1 in the original publication are correct.

A Turing instability in $\left(u_{*}, v_{*}\right)$ requires that (1), (2) are stable in this point under spatially homogeneous perturbations. The additional assumption (5) corresponds to the stability of the ODE reduction also in directions of spatially heterogeneous perturbations. This property automatically holds in the case of a local reaction-diffusion system. In case of the non-local system (1), (2) however, (5) is not necessarily true and might not be a biologically relevant condition. Therefore the corrected statement still allows for the possibility of diffusive instabilities in (1), (2), unlike the conclusions of original paper.

All other results of the original publication remain true, even though the authors use in Theorem 3.4 a condition for Turing instabilities that in the present context is not optimal. 